\title{
A thyroglossal cyst with endolaryngeal extension: A Case Report
}

\section{Case Report}

\author{
Ahmed Hammad ${ }^{1}$,Hazem M. Abdel Tawab ${ }^{1,2}$, Biji Varghese ${ }^{1}$, Ravi Kumar V. ${ }^{1}$, Ibrahim \\ A. Sulaiman ${ }^{3}$
}

${ }^{1}$ Department of Otorhinolaryngology, Sultan Qaboos Hospital, Oman

${ }^{2}$ Department of Otorhinolaryngology, Faculty of Medicine, Cairo University, Egypt

${ }^{3}$ Department of Pathology, Sultan Qaboos Hospital, Oman

\begin{abstract}
Background Thyroglossal duct cyst is the most common midline congenital swelling in the head and neck. This anomaly is very rare in elderly. It usually presents as a midline painless neck swelling. In rare cases, it may show abnormal extension into the larynx that manifests with dysphonia and laryngeal obstruction. Case report We report a case of thyroglossal cyst in a sixty five year old patient who presented with difficulty in breathing, and hoarseness of voice. Fiber optic laryngoscopy revealed fullness in the right vallecula and the right piriform fossa. The differential diagnosis on Ultrasound and Computed Tomography was thyroglossal cyst versus laryngocele. After surgical excision, dyspnea resolved and voice improved. Postoperative fiber optic laryngoscope revealed the disappearance of previous fullness. Histopathological examination confirmed the diagnosis of thyroglossal cyst. To the best of our knowledge, very few cases of thyroglossal cyst with laryngeal extension were encountered in literature, and in this study, we are reporting another case of this extremely rare condition.
\end{abstract}

Key Words: Hoarseness of voice, larynx, thyroglossal cyst

Received: 19 December 2018, Accepted: 07 January 2019

Corresponding Author: Hazem M. Abdel Tawab, MD, Department of Otorhinolaryngology, Faculty of Medicine, Cairo University, Egypt, Department of Otorhinolaryngology, Sultan Qaboos Hospital, Oman, Tel.: 0096891128430, E-mail: hazemabdeltawwab77@yahoo.com

ISSN: 2090-0740, March 2019, Vol.20, No.1

\section{INTRODUCTION}

Thyroglossal duct cyst (TDC) develops from remnants of the thyroglossal duct and presents in the midline in relation to the hyoid bone. It enlarges as a result of inflammation, infection, or mucus retention ${ }^{[1]}$ It is rare in elderly patients with a reported incidence of approximately $0.6 \%$ in the $6^{\text {th }}$ decade $^{[2]}$ It usually presents as painless midline neck swelling that moves with protrusion of tongue and deglutition and, intimately related to the hyoid bone $^{[3]}$ Although thyroglossal cysts are typically benign and asymptomatic, some have been shown to contain malignancy, cause airway obstruction, or extend into the larynx ${ }^{[4]}$ Most of the reported cases occurred in elderly males where few of them were with no obvious neck mass.

\section{Case report}

A sixty five year old male presented to the accident and emergency with difficulty in breathing and change in voice. He was admitted by the respiratory physicians with a suspicion of respiratory failure and was initially treated in the Intensive Care Unit with CPAP (Continuous Positive Airway Pressure), antibiotic, steroid and bronchodilator medications which resulted in mild improvement. An ENT opinion was sought. The patient gave a history of a painless swelling in the neck, which increased in size slowly over the past 5 years, change in voice of one month duration and increasing dyspnea of 10 days duration. There was no history of recent rapid increase in size of the swelling. There was no history suggesting any chronic illness, prior surgery, or trauma.

On examination, the patient was in respiratory distress receiving oxygen by nasal prongs at a rate of 6 liters per minute to maintain saturations above $90 \%$. He had a hot potato voice. Neck examination revealed a $5 \mathrm{~cm} \times 3 \mathrm{~cm}$ mass in the anterior neck extending from the level of hyoid bone superiorly to the level of the thyroid cartilage inferiorly, had smooth surface and cystic consistency. The mass was moving with deglutition but not with protrusion of the tongue. The skin was not attached to the mass. There was no cough impulse and it did not become prominent with Valsalva maneuver. Fiber-optic laryngoscopy revealed fullness and cystic swelling in the right vallecula and the right piriform fossa with deviation of the right side of the supraglottis medially and the epiglottis almost touching the posterior pharyngeal wall (Figure 1). The left cord was normally mobile while the right vocal cord was obscured by the fullness. 


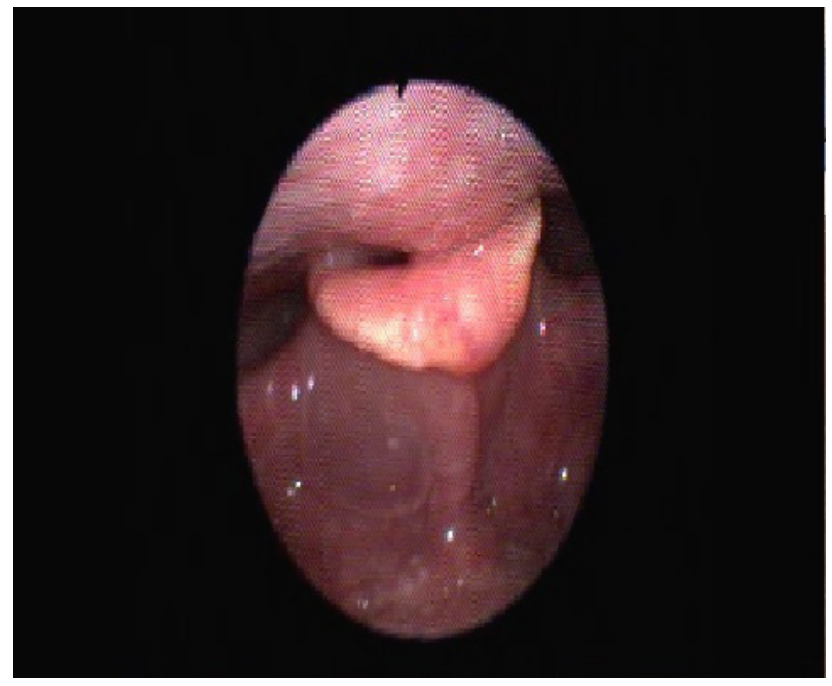

Fig. 1: Preoperative fiber-optic laryngoscopy revealed fullness and cystic swelling in the right vallecula.

Ultrasound revealed a cystic lesion seen in the middle part of the right anterior aspect of the neck measuring about $5.5 \times 3.4 \mathrm{~cm}$ showing fine internal echoes (complicated cyst). Contrast CT neck scan revealed a non-enhancing mass $5.1 \times 4.2 \times 3 \mathrm{~cm}$ situated between the upper edge of thyroid cartilage and the hyoid bone with an intralaryngeal extension. Approximately half of it is inside the airway, causing partial compression of the right side of the supraglottis (Figure 2). The remaining portion is bulging outside and is of high density fluid 20-25 cc. The thyroid gland was found to be in its normal position. Based on the clinical history with examination findings of a cystic swelling that moves up and down with deglutition and no impulse on cough with no enhancement in the CT scan, a suspicion of a thyroglossal cyst was raised and surgery decision was taken.

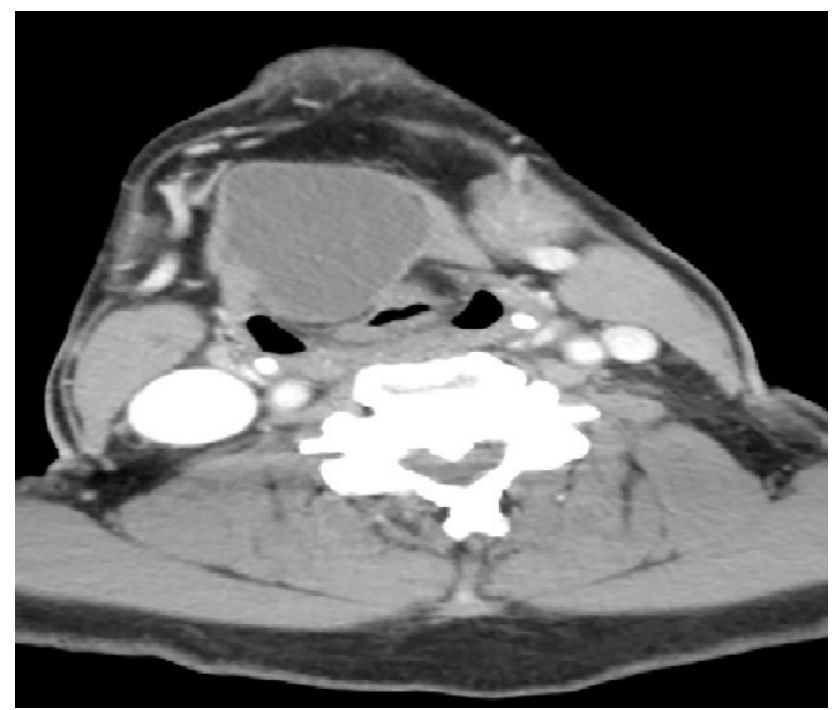

Fig. 2: Contrast CT neck showing non-enhancing mass causing partial compression of the right side of the supraglottis.
The necessity, nature and complications of surgery were explained to the patient and informed consent was taken. Consent was also taken for tracheostomy under local anesthesia in the event of failed intubation. Through size 6 endotracheal tube the airway was secured and anesthesia administered. Sistrunk's operation was done using standard technique. Intraoperatively, the mass was seen extending more to the right side. The intralaryngeal component was identified, dissected free of the laryngeal mucosa and delivered into the neck (Figure 3). The cyst was dissected thoroughly till the hyoid bone where the central part of hyoid bone was excised to avoid recurrence. The patient was extubated and observed overnight in the intensive care unit. The post-operative period was uneventful with complete resolution of dyspnea and improvement of voice. He was discharged on $8^{\text {th }}$ post op day as he lived in a remote area. Post-operative fiber optic laryngoscopy showed resolution of the fullness and cystic swelling in the right vallecula and the right piriform fossa and normal positioning of the larynx (Figure 4). Histopathological examination described the cyst as: Grossly; the cyst appeared well encapsulated. The external surface was nearly smooth, with attached fibroadipose tissue. The size of the cyst was $5.5 \mathrm{~cm}$ in maximum diameter. Sectioning revealed unilocular cyst filled with thick yellowish gelatinous material. (Figure 5). Microscopic examination showed that the cyst wall was made of dense fibrous tissue with granulation tissue. The cyst wall failed to disclose any epithelial lining. Cholesterol crystal clefts, anucleate cells, and cell debris were seen in the cyst content. Lobules of variably sized colloid containing thyroid follicles with foci of reactive lymphoid tissues were seen in the subjacent stroma about the cyst wall. (Figure 6).

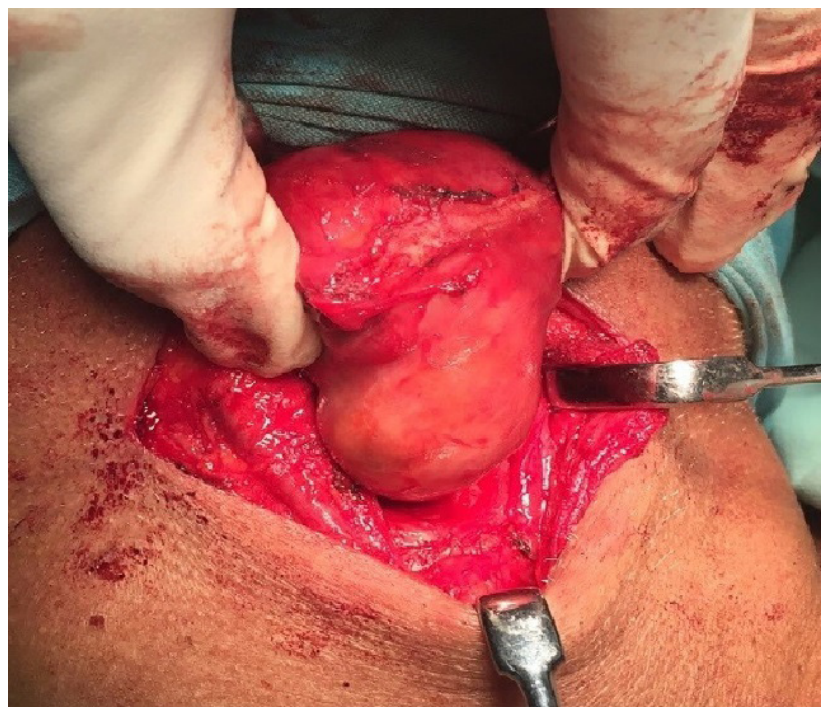

Fig. 3: The intralaryngeal component of the cyst dissected and delivered into the neck. 


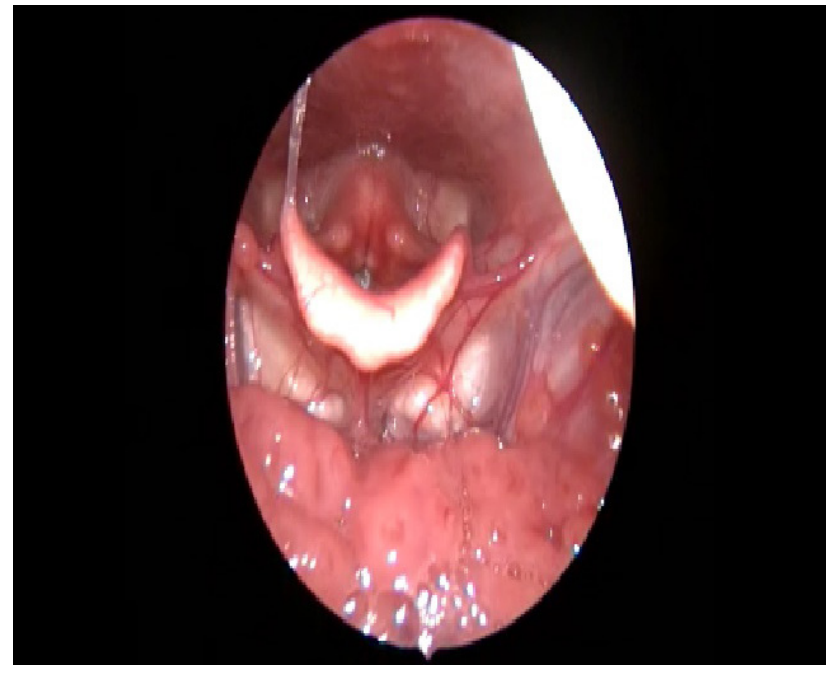

Fig. 4: Postoperative fiber optic laryngoscope revealed disappearance of fullness and cystic swelling in the right vallecula.

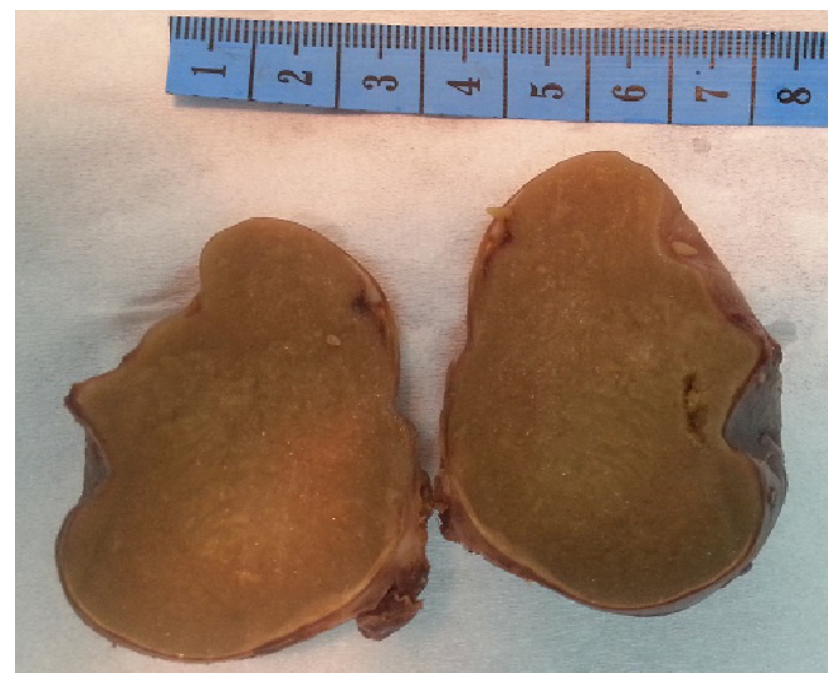

Fig. 5: Thyroglossal duct cyst. The content of the cyst is gelatinous.

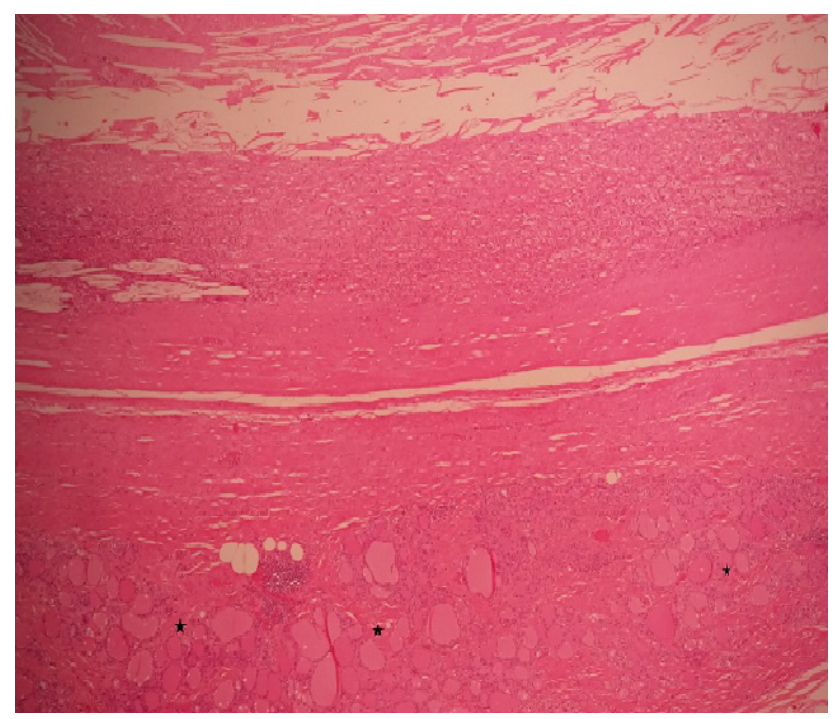

Fig. 6: Fibrous wall, absent epithelium and granulation tissue with cholesterol crystal clefts. Anucleate cells, and cell debris present. Colloid containing thyroid follicles (stars) can be seen in the stroma. (H\&E, x40).

\section{DISCUSSION}

TDC is the most common congenital anomaly of the neck in childhood, representing more than $75 \%$ of congenital midline neck masses. Although TDCs often occur in pediatric patients, at least half are diagnosed in the second decade of life and they can also present later in adulthood. TDCs originate from persistent epithelial remnants of the thyroglossal duct that are present during the descent of the thyroid gland from the foramen cecum to its final position in the anterior neck ${ }^{[5]}$

TDC usually presents as a painless midline neck swelling that moves with deglutition and protrusion of the tongue with the typical radiological finding of a simple cystic swelling in the neck. Occasionally, TDC can show an atypical presentation either clinically or radiologically, which may pose a diagnostic challenge. Failure to anticipate the possibility of a TDC may be associated with the performance of an inadequate surgical procedure such as simple incisional biopsy or enucleation, both of which are associated with significant recurrence rates ${ }^{[6]}$

In this report we want to highlight one atypical presentation and atypical extension of thyroglossal cyst in a sixty five years old male who had difficulty in breathing and change of voice and showed abnormal extension into the larynx. According to Kurien et al in 2012, they mentioned 15 cases of thyrogloassal cyst with laryngeal extension ${ }^{[7]} \mathrm{Few}$ other cases with endolaryngeal extension had been mentioned in literature from 2014 till October 2018 ${ }^{[8-12]}$ Discrepancies about the exact number of cases of thyroglossal cyst with laryngeal extension were found during our literature search which might be related to miscounting of all cases from all scientific search engines. To the best of our knowledge, our case presented here is approximately number twenty three based on all found material in literature search.

Challenges in diagnosis include: exclusion of other laryngeal cysts such as saccular cysts and laryngoceles. Saccular cysts are submucosal and result from fluid collection in the laryngeal saccule due to saccular orifice occlusion. Laryngoceles are air-filled, submucosal out pouching of laryngeal mucosa that is communicated directly to the laryngeal inlet ${ }^{[13]}$ Thyroglossal duct cysts originate outside of the larynx, while saccular cysts and laryngoceles originate inside. However, their appearance in fiber optic laryngoscope and neck CT are very similar, making differential diagnosis difficult. While thyroglossal duct cyst fiberscope findings are similar to those associated with laryngeal cysts, radiological examinations, particularly CT or magnetic resonance imaging (MRI), are helpful in diagnosis ${ }^{[14]}$

The treatment of choice for (TDC) is a Sistrunk's procedure in which a portion of the hyoid bone is removed by meticulous excision of persistent duct up to foramen caecum to reduce recurrence ${ }^{[15]}$ 


\section{CONCLUSION}

We should keep in mind the atypical presentations and atypical extensions of thyroglossal cyst even in absence of neck mass. Thyroglossal cyst with laryngeal extension may mimic laryngeal cysts, but CT scan and histopathological assessment are crucial in diagnosis.

\section{CONFLICT OF INTEREST}

The authors declare no conflicts of interest

\section{FINANCIAL DISCLOSURE}

No financial support to this study.

\section{REFERENCES}

1. Kurt A, Ortug C, Aydar Y, Ortug G. An incidence study on thyroglossal duct cysts in adults. Saudi Med J. 2007; 28:593-597.

2. Filho FW, Costa FW, Turatti E, Cavalcante RB. Extensive thyroglossal duct cyst in 83-year-old patient. N Y State Dent J. 2012; 78:39-41.

3. Iwata T, Nakata S, Tsuge H, Koide F, Sugiura M, Otake $\mathrm{H}$, et al. Anatomy-based surgery to remove thyroglossal duct cyst: two anomalous cases. J Laryngol Otol. 2010; 124:443-446.

4. Lubben B, Alberty J, Lang-Roth R, Seifert E, Stoll $\mathrm{W}$. Thyroglossal duct cyst causing intralaryngeal obstruction. Otolaryngol Head Neck Surg. 2001; 125:426-427.

5. Mondin V, Ferlito A, Muzzi E, Silver CE, Fagan JJ, Devaney KO, et al .Thyroglossal duct cyst: personal experience and literature review. Auris Nasus Larynx. 2008; 35:11-25.
6. Ducic Y, Chou S, Drkulec J, Ouellette H, Lamothe A. Recurrent thyroglossal duct cysts: a clinical and pathologic analysis. Int J Pediatr Otorhinolaryngol. 1998; 44:47-50.

7. Kurien R, Michael RS. Subglottic thyroglossal duct cyst: A rare intralaryngeal presentation. Ear Nose Throat J. 2012; 91 (7): E15: 9.

8. Verma H, Dass A, Singha SK, Gupta N, Punia RPS Thyroglossal duct cyst: An intralaryngeal extension. J Diseases and disorders. 2014; 1 (2): 005-009.

9. Aslier M, Ikiz A, Erdag T. Thyroglossal duct cyst with endolaryngeal extension. Turk Arch Otolaryngol. 2014; 52: 112-114.

10. Hannah $\mathrm{Ng}$ Jia Hui and Gerald Tay Ci-An. VideoEndocrinology. 2016; 3(2).

11. Masako Shimizu, Fumiaki Matsumi, Mika Tsuruoka Endolaryngeal extension of laterally located thyroglossal duct cyst. Nihon Kikan Shokudoka Gakkai Kaiho. 2016; 67(4):303-308.

12. Tomoaki Murakami, Daisuke Maki, Koji Ebisumoto, Akihiro Sakai, Masahiro Iida, Kenji Okami. A case of thyroglossal cyst with laryngeal extension. J Japan Soc Head Neck Surg. 2018; 28(1): 91-94.

13. Sniezek JC, Johnson RE, Ramirez SG, Hayes DK. Laryngoceles and saccular cysts. South Med J. 1996; 89: 427-430.

14. Cumberworth VL, Bradley PJ. Atypical thyroglossal duct cyst. J Laryngol Otol. 1989; 103:700-703.

15. Narayana Moorthy S, Arcot R. Thyroglossal duct cyst-more than just an embryological remnant. Indian J Surg. 2011; 73:28-31. 